J. Dairy Sci. 96:6877-6882

http://dx.doi.org/10.3168/jds.2013-6881

(c) American Dairy Science Association ${ }^{\circledR}, 2013$.

\title{
Microwave-assisted extraction and determination of dicyandiamide residue in infant formula samples by liquid chromatography-tandem mass spectrometry
}

\author{
Yan Shen, ${ }^{* 1}$ Chao Han, $†$ Xiujin Zhou, $\ddagger$ Xiangzhun Chen, $†$ Fuzhen Huang, $†$ and Zhenou Zhu $†$ \\ ${ }^{*}$ College of Chemistry and Materials Engineering, Wenzhou University, Wenzhou 325035, China \\ †Wenzhou Entry-Exit Inspection and Quarantine Bureau of P.R.C, Wenzhou 325027, China \\ $\ddagger Z$ houshan Entry-Exit Inspection and Quarantine Bureau of P.R.C, Wenzhou 325027, China
}

\begin{abstract}
A simple, precise, accurate, and validated liquid chromatography-tandem mass spectrometry (LC-MS/ MS) method was developed for the determination of dicyandiamide residue in infant formula samples. Following microwave-assisted extraction with $5 \%$ formic acid and clean-up on a Sep-Pak AC-2 SPE cartridge, samples were separated on a ZIC-HILIC HPLC column $(150 \times 2.1 \mathrm{~mm}$ i.d., $5-\mu \mathrm{m}$ film thickness; Merck KGaA, Darmstadt, Germany) with $20 \mathrm{~m} M$ ammonium acetate solution-acetonitrile as mobile phase at a flow rate of $0.25 \mathrm{~mL} / \mathrm{min}$. A linear calibration curve was obtained in the concentration range from 1.0 to $50 \mathrm{ng} / \mathrm{mL}$. Infant formula samples were fortified with dicyandiamide at 3 levels, producing average recovery yields of 83.6 to 95.7\%. The limits of detection and quantification of dicyandiamide were 3 and $10 \mu \mathrm{g} / \mathrm{kg}$, respectively. Due to its simplicity and accuracy, the straightforward method is particularly suitable for routine dicyandiamide detection.
\end{abstract}

Key words: dicyandiamide, infant formula, microwave-assisted solvent extraction, liquid chromatography-tandem mass spectrometry

\section{INTRODUCTION}

Dicyandiamide (DCD), or cyanoguanidine, is produced on a large scale from Nitro-chalk via the intermediate compound cyanamide in a 2 -step process. In addition to its various industrial applications, its potential as a nitrification inhibitor is of high importance in agriculture (Schwarzer and Haselwandter, 1996). The nitrification inhibitor DCD reduces the rate of nitrification in soils, subsequently limiting $\mathrm{N}$ losses. Dicyandiamide's bacteriostatic mode of inhibiting

Received April 1, 2013.

Accepted July 27, 2013.

${ }^{1}$ Corresponding author: shenyan@wzu.edu.cn ammonia-oxidizing bacteria has raised concerns about the efficacy of frequent DCD use (Clough et al., 2007; Wakelin et al., 2013). Because of residual DCD on pastures and dairy cow eating these pastures, it has been reported that DCD was detected in infant formula. Thus, a sensitive method to measure residue levels of DCD in infant formula is needed to detect and evaluate whether low levels of this compound are present in products intended for infant consumption.

Dicyandiamide has been analyzed by various methods, including Raman chemical imaging (Qin et al., 2013), liquid chromatography-tandem mass spectrometry (LC-MS/MS; MacMahon et al., 2012), and LC (Nagumo et al., 2009). Very few reports exist about the determination of DCD in infant formula.

Currently, the application of advanced LC-MS/MS to analyze residues has allowed a broad range of compounds to be determined, and this has permitted the comprehensive assessment of food contaminants. Low maximum residue limits have fostered the development of more powerful and sensitive analytical methods to meet the requirements of complex samples, such as food. In this sense, LC-MS/MS with a triple quadrupole in multiple reaction monitoring mode has become the most widely used technique for the quantitation of (polar) residues in food, as reported extensively in the literature.

For isolation of the DCD, microwave-assisted solvent extraction (MAE) seems particularly attractive, because of the rapid heating of aqueous samples (Han et al., 2011). The principal of the method is that microwave energy is absorbed by the extractant, which in turn transfers it to the sample in the form of heat. Partitioning of the analytes from the sample matrix to the extractant depends mainly on the temperature and the nature of the extractant. Unlike classical heating, microwaves heat the entire sample simultaneously (Camel, 2000). Microwave-assisted solvent extraction can reduce both extraction time and solvent consumption compared with conventional methods. Many cases 
have already proven that MAE is a viable alternative to conventional techniques for many kinds of samples (Wang et al., 2012).

The present study proposes a method for the analysis of DCD in infant formula by LC-MS/MS. The method was then used to analyze 100 infant formula samples purchased from local supermarkets.

\section{MATERIALS AND METHODS}

\section{Chemicals, Reagents, and Equipment}

All reagents and solvents were analytical grade unless otherwise specified. A certified DCD standard (99\% purity) was purchased from National Institutes for Food and Drug Control (Beijing, China); a ${ }^{15} \mathrm{~N}_{4}$-labeled DCD $\left({ }^{15} \mathbf{N}_{4}\right.$-DCD) standard was supplied by Toronto Research Chemicals Inc. (North York, ON, Canada). The structures of DCD and ${ }^{15} \mathrm{~N}_{4}$-DCD are shown in Figure 1. The water used was purified with a Milli-Q water purification system (Millipore Corp., Bedford, MA); HPLC-grade dichloromethane, acetonitrile, and methanol were obtained from Merck KGaA (Darmstadt, Germany). Formic acid (99\% purity) was from Sigma-Aldrich Chemie GmbH (Steinheim, Germany). Ammonium acetate was acquired from Alfa Aesar (Ward Hill, MA). An MAE system (Ethos; Milestone Systems, Wiesbaden, Germany) and LC-MS/MS (Agilent 1200-API 4000; Agilent Technologies Inc., Santa Clara, CA) were used in sample analysis. The LC and MS/MS analytical conditions are given in Tables 1 and 2 , respectively.

\section{Preparation of Standards}

A stock standard solution of DCD was prepared at concentration of $100 \mu \mathrm{g} / \mathrm{mL}$ in acetonitrile, stored in a refrigerator at $4^{\circ} \mathrm{C}$ in the dark, and used to prepare working standard solutions by appropriate dilution with acetonitrile. Each standard solution was injected into the LC-MS/MS system 3 times. A specific average peak area was regressed with a specific level to calculate the calibration equation.
(A)

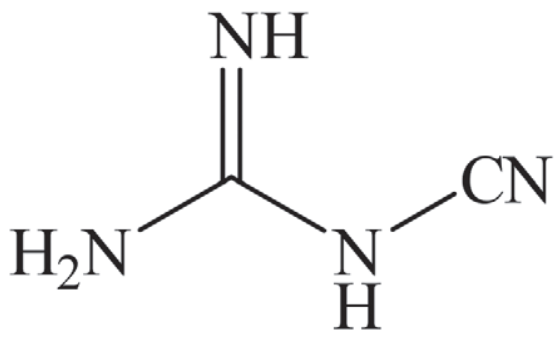

(B)

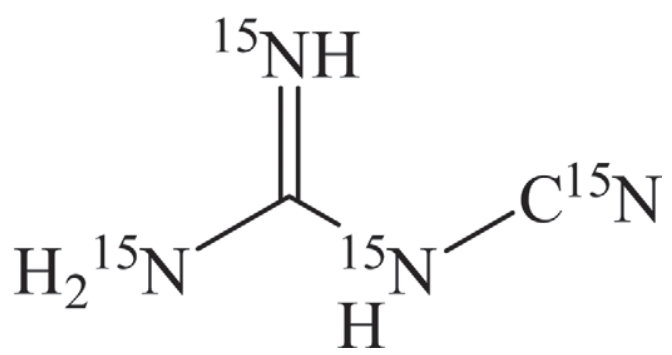

Figure 1. Chemical structure of (A) dicyandiamide (DCD) and (B) ${ }^{15} \mathrm{~N}_{4}$-labeled DCD $\left({ }^{15} \mathrm{~N}_{4}\right.$-DCD).

\section{Sample Preparation}

Microwave-assisted extraction was performed with an Ethos MAE system (Milestone Systems) equipped with a 12-vessel carousel, operated in the closed-vessel mode, and a magnetic stirrer. Polytetrafluoroethylene (PTFE)-lined extraction vessels were used and during operation both temperature and pressure were monitored; a fiber optic temperature sensor in the interior of the microwave oven was also used. An accurately weighed $1.00 \mathrm{~g}$ powdered sample was placed into an extraction vessel. Next, $500 \mu \mathrm{L}$ of ${ }^{15} \mathrm{~N}_{4}$-DCD (1,000 $\mu \mathrm{g} /$ $\mathrm{mL}$ ) and $20 \mathrm{~mL}$ of $5.0 \%$ formic acid solution were placed in an extraction vessel and the vessel was capped. On the basis of a predesigned experimental trial, the temperature was increased to $80^{\circ} \mathrm{C}$ from room temperature in $5 \mathrm{~min}$ and then kept constant at $80^{\circ} \mathrm{C}$ for $25 \mathrm{~min}$.

Table 1. Analytical conditions for liquid chromatography

\begin{tabular}{|c|c|}
\hline Item & Details \\
\hline Instrument & Agilent 1200 system (Agilent, Germany) \\
\hline Column & ZIC HILIC HPLC column $(150 \times 2.1-\mathrm{mm}$ i.d., $5-\mu \mathrm{m}$ film thickness; Merck KGaA, Darmstadt, Germany) \\
\hline Mobile phase & $\begin{array}{l}\mathrm{A}=20 \mathrm{mM} \text { ammonium acetate solution; } \mathrm{B}=\text { acetonitrile; gradient elution used with the ratio of } \mathrm{A}: \mathrm{B} \text { varied as } \\
\text { follows: } 0 \mathrm{~min}, 10: 90 ; 6 \mathrm{~min}, 10: 90 ; 6.1 \mathrm{~min}, 40: 60 ; 12 \mathrm{~min}, 40: 60 ; 12.1 \mathrm{~min}, 10: 90 ; 20 \mathrm{~min}, 10: 90 \text {. Flow was diverted to } \\
\text { waste from } 0 \text { to } 3.5 \mathrm{~min} \text { and from } 6.0 \text { to } 20 \mathrm{~min}\end{array}$ \\
\hline Flow rate & $0.25 \mathrm{~mL} / \mathrm{min}$ \\
\hline Column temperature & $30^{\circ} \mathrm{C}$ \\
\hline
\end{tabular}


Table 2. Analytical conditions for tandem mass spectrometry (MS/MS; API 4000; Applied Biosystems, Burlington, ON, Canada)

\begin{tabular}{|c|c|c|c|c|c|}
\hline \multirow[b]{2}{*}{ Item } & \multicolumn{5}{|c|}{ Analytical conditions ${ }^{1}$} \\
\hline & Retention time (min) & $\begin{array}{l}\text { Q1 mass } \\
\text { (mass no.) }\end{array}$ & $\begin{array}{l}\text { Q3 mass } \\
\text { (mass no.) }\end{array}$ & $\mathrm{CE}(\mathrm{eV})$ & $\mathrm{DP}(\mathrm{V})$ \\
\hline \multicolumn{6}{|l|}{ Compound } \\
\hline \multirow{2}{*}{ Dicyandiamide (DCD) } & 4.82 & 85.0 & $68.0^{2}$ & 22 & 50 \\
\hline & & 85.0 & 43.0 & 18 & 50 \\
\hline \multirow[t]{2}{*}{${ }^{15} \mathrm{~N}_{4}-\mathrm{DCD}$} & 4.82 & 89.0 & $71.0^{2}$ & 25 & 52 \\
\hline & & 89.0 & 45.0 & 18 & 55 \\
\hline Mass spectrometry & \multicolumn{5}{|c|}{ Optimized value } \\
\hline Ionization & \multicolumn{5}{|c|}{ ESI positive mode ${ }^{3}$} \\
\hline Source temperature $\left({ }^{\circ} \mathrm{C}\right)$ & \multicolumn{5}{|c|}{500} \\
\hline Ionization voltage (V) & \multicolumn{5}{|c|}{5,500} \\
\hline Ion source $(\mathrm{GS} 1)$ setting ${ }^{4}$ & \multicolumn{5}{|c|}{45} \\
\hline Ion source (GS2) setting ${ }^{4}$ & \multicolumn{5}{|c|}{45} \\
\hline Curtain gas setting & \multicolumn{5}{|c|}{15} \\
\hline CAD gas setting ${ }^{5}$ & \multicolumn{5}{|c|}{10} \\
\hline Dwell time & \multicolumn{5}{|c|}{$100 \mathrm{~ms}$} \\
\hline
\end{tabular}

The power of MAE was $800 \mathrm{~W}$. After extraction, the vessels were cooled to room temperature before opening. The suspension was centrifuged at $8,875 \times g$ for 15 min. The supernatant layer was then transferred into a $25-\mathrm{mL}$ volumetric flask and diluted with $5.0 \%$ formic acid solution.

\section{Solid-Phase Extraction}

Extracts were cleaned by solid-phase extraction on a Sep-Pak Plus AC-2 cartridge (400 mg, $6 \mathrm{~mL}$; Waters Corp., Milford, MA). After conditioning of the cartridge with acetonitrile $(10 \mathrm{~mL})$ and then with $10 \mathrm{~mL}$ of water, the sample $(5 \mathrm{~mL})$ was applied and left to flow through under the action of gravity. The cartridge was washed with $10 \mathrm{~mL}$ of water, dried by application of vacuum for $1 \mathrm{~min}$, and then eluted into a glass culture tube with $30 \mathrm{~mL}$ of acetonitrile/ methanol $(3 / 2, \mathrm{vol} / \mathrm{vol})$. The eluate was evaporated to dryness under vacuum at $45^{\circ} \mathrm{C}$. The dried extract was reconstituted in $2.0 \mathrm{~mL}$ of acetonitrile, vortex mixed for $60 \mathrm{~s}$, and filtered through a $0.22-\mu \mathrm{m}$ filter into a glass LC vial.

\section{RESULTS AND DISCUSSION}

\section{Optimization of Extraction}

The method of extraction, extraction solvent, and extraction time were investigated to obtain reasonable experimental results and satisfactory efficiency. The efficiency of extraction of DCD by MAE was compared with that obtained by ultrasonic extraction. Recoveries of DCD from infant formula samples were 92.4 to $95.6 \%$ (MAE) and 79.3 to $84.5 \%$ (ultrasonic extraction). Recoveries using MAE were 11.1 to $13.1 \%$ higher than recoveries using ultrasonic extraction, so MAE was used in the experiments. According to the literature (Nagumo et al., 2009), 2.0\% formic acid and 5.0\% acetic acid solutions have been used for extraction solvent selection experiments. On the basis of a predesigned experimental trial, the temperature was increased to $80^{\circ} \mathrm{C}$ in 5 min and then kept constant at $80^{\circ} \mathrm{C}$ for 25 min. The results showed that $5.0 \%$ formic acid solution was the most effective solvent. Samples were, therefore, extracted with $5.0 \%$ formic acid solution by MAE for 15,30 , and $45 \mathrm{~min}$ to find the optimum extraction time. Recoveries of DCD from infant formula samples were 75.8 to $77.3 \%$ (15 $\mathrm{min}$ ), 90.4 to $92.6 \%$ (30 $\mathrm{min}$ ), and 90.8 to $92.5 \%$ (45 min). Recoveries using $30 \mathrm{~min}$ extraction were similar to recoveries using $45 \mathrm{~min}$ extraction and 14.6 to $15.3 \%$ higher than recoveries using 15 min extraction. Therefore, all subsequent extractions were performed using one 30-min cycle. Because temperature is an important experimental condition in MAE, extractions were performed at 60,80 , and $100^{\circ} \mathrm{C}$. Recoveries of DCD from infant formula samples were 83.8 to $87.2 \%\left(60^{\circ} \mathrm{C}\right), 92.1$ to $94.3 \%\left(80^{\circ} \mathrm{C}\right)$, and 91.6 to $93.5 \%\left(100^{\circ} \mathrm{C}\right)$. Recoveries at $80^{\circ} \mathrm{C}$ were similar to recoveries at $100^{\circ} \mathrm{C}$ and 7.1 to $8.3 \%$ higher than recoveries at $60^{\circ} \mathrm{C}$. Therefore, all subsequent extractions were performed at $80^{\circ} \mathrm{C}$. 


\section{Matrix Effects}

Matrix effects (generally recognized as a suppression or enhancement of the analytical signal due to coeluting matrix components) have been widely studied and recognized as a source of error in quantitative LC-MS/MS analysis of food samples (Niessen et al., 2006). In the current study, we chose to use ${ }^{15} \mathrm{~N}_{4}$-DCD as an internal standard to account and correct for matrix effects. The use of matrix-matched calibration standards was done to compensate for the matrix effect (i.e., signal suppression or enhancement of studied pesticides in matrix solution). The matrix effect, expressed as the signal from the DCD in the matrix compared with the signal in the solvent was tested in infant formula sample matrices. Dicyandiamide was significantly affected by the matrix components and the recoveries calculated using the solvent calibration curve were 64.2 to $72.4 \%$. In contrast, the recoveries calculated using the matrix solution calibration curve were 91.3 to $94.2 \%$. Table 3 shows matrix effects for DCD at the concentration level of $200 \mu \mathrm{g} / \mathrm{kg}$ in infant formula samples. Recoveries using the matrix solution calibration curve were 21.8 to $27.1 \%$ higher than recoveries using the solvent calibration curve. Therefore, for an accurate quantification, the use of matrix solution calibration curve is required.

\section{Method Validation}

Linearity of Calibration Standards. The concentrations of the analyte in the samples were calculated by matrix calibration using the internal standard. It was evident from the results that more reproducible results were obtained using ${ }^{15} \mathrm{~N}_{4}$-DCD as the internal standard for quantification of analyzed substances. Calibration curve with $1 / \mathrm{x}$ weighting was plotted for the analyte. The matrix calibration curve was linear over the range of 1 to $50 \mathrm{ng} / \mathrm{mL}$ for the analyzed compound. The correlation coefficient was 0.9996 . The limits of detection and quantification of dicyandiamide were 3 and $10 \mu \mathrm{g} /$ $\mathrm{kg}$, respectively. Quantification was performed based

Table 3. Infant formula matrix effects for dicyandiamide (DCD)

\begin{tabular}{lcc}
\hline $\begin{array}{l}\text { Sample } \\
\text { no. }\end{array}$ & $\begin{array}{c}\text { Recovery }^{1} \\
(\%)\end{array}$ & $\begin{array}{c}\text { Recovery }^{2} \\
(\%)\end{array}$ \\
\hline 1 & 64.2 & 92.5 \\
2 & 68.5 & 93.6 \\
3 & 71.3 & 91.7 \\
4 & 72.4 & 94.2 \\
5 & 70.5 & 93.8 \\
6 & 67.3 & 91.3 \\
\hline
\end{tabular}

${ }^{1}$ Solvent calibration curve.

${ }^{2}$ Matrix solution calibration curve. on calibration plots using the peak area of the most intense transition of the analyte.

Assay Specificity. The specificity was evaluated by the analysis of 10 blank samples of different infant formula samples. No interfering peaks from endogenous compounds were found in the retention time of the target analyte for infant formula samples.

Precision and Accuracy. Intraday precision was examined by analysis of the same standard solution at 3 different concentrations (high, medium, and low) on the same day; interday precision was determined by analysis of the same solutions on 3 different days. Relative standard deviation values were 2.24 and $2.63 \%$, respectively. Method accuracy was evaluated by recovery studies, using a "blank" infant formula sample labeled as organically produced and confirmed as not containing the target DCD compound using the proposed method. "Blank" infant formula samples were spiked with the appropriate amounts of the target compound at 3 concentration levels: 10, 50, and $200 \mu \mathrm{g} / \mathrm{kg}$. The DCD content was determined by use of the corresponding calibration plot and recovery was calculated from the ratio of the amounts detected to those added. Average recovery was in the range of 83.6 to $95.7 \%$ (Table 4) and with relative standard deviations below $6.2 \%$, indicative of the good recovery and precision of the method.

Real Samples. Initial chromatographic experiments were carried out to verify the possibility of proposing an LC-MS/MS method for the determination of DCD. When the chromatographic conditions were optimized, DCD was well retained (retention time $=4.82 \mathrm{~min}$ ) and eluted with good peak shape. Initial extraction experiments using DCD-spiked samples demonstrated the selectivity of the method. Figure 2 shows a chromatogram corresponding to 1 spiked sample, where a clean background was obtained, demonstrating the absence of chromatographic interferents. Figure 3 shows the

Table 4. Mean recoveries and repeatability of the developed method at 3 concentration levels ${ }^{1}$

\begin{tabular}{lccc}
\hline $\begin{array}{l}\text { Sample } \\
\text { no. }\end{array}$ & $\begin{array}{c}\text { Dicyandiamide } \\
\text { spiking level } \\
(\mu \mathrm{g} / \mathrm{kg})\end{array}$ & $\begin{array}{c}\text { Recovery } \\
(\%)\end{array}$ & $\begin{array}{c}\mathrm{RSD}^{2} \\
(\%)\end{array}$ \\
\hline 1 & 10 & 84.9 & 5.7 \\
& 50 & 88.5 & 4.6 \\
2 & 200 & 94.5 & 3.1 \\
& 10 & 83.6 & 6.2 \\
& 50 & 87.3 & 4.8 \\
& 200 & 93.2 & 3.3 \\
& 10 & 86.2 & 5.5 \\
& 50 & 89.3 & 4.3 \\
& 200 & 95.7 & 2.8 \\
\hline
\end{tabular}

${ }^{1}$ The limit of quantification for all samples was $10 \mu \mathrm{g} / \mathrm{kg}(\mathrm{n}=6)$. ${ }^{2}$ Relative SD. 


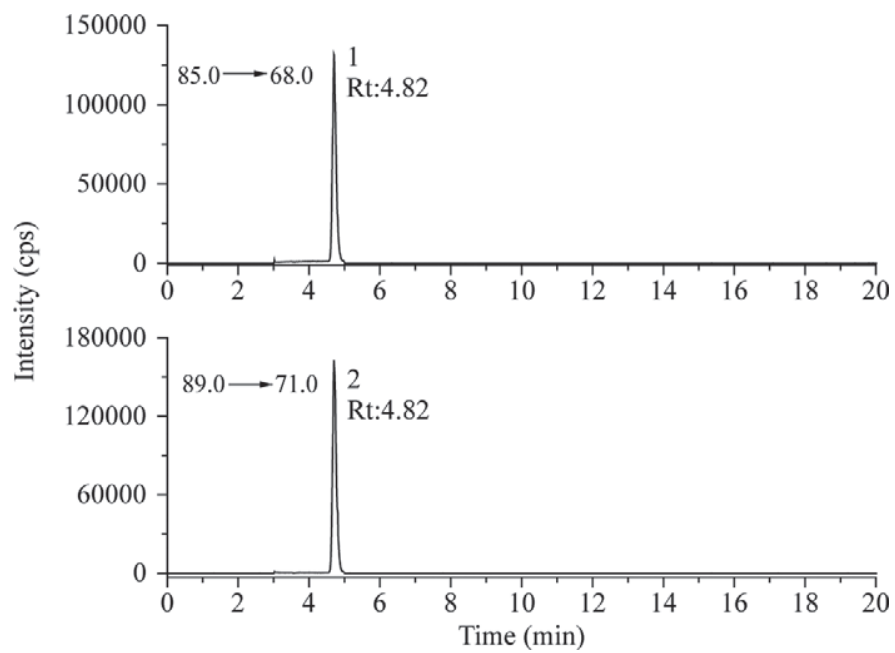

Figure 2. Liquid chromatography-tandem mass spectrometry (LC-MS/MS) multiple reaction monitoring (MRM) chromatograms of spiked samples containing (1) dicyandiamide (DCD) and $(2)^{15} \mathrm{~N}_{4^{-}}$ labeled DCD $\left({ }^{15} \mathrm{~N}_{4}\right.$-DCD). Rt $=$ retention time $(\mathrm{min})$.

full scan mass spectra of DCD and ${ }^{15} \mathrm{~N}_{4}$-DCD. Onehundred infant formula samples were purchased from local supermarkets and analyzed following the proposed method; no DCD was confirmed in all samples. The consumption of these foods does not constitute a health risk for consumers.

\section{CONCLUSIONS}

The MAE extraction provides an efficient and reliable method for the quantitative recovery of DCD. Compared with use of ultrasonic extraction, the MAE method is a highly promising technology for extraction of DCD from infant formula samples using 5.0\% acetic acid solution as extraction solvent. DCD residue spiked in infant formula samples was effectively separated from matrix components in MAE extracts by using Sep-Pak Plus AC-2 solid-phase extraction cartridge and a ZIC HILIC column. A sensitive LC-MS/ MS method to analyze DCD residues in infant formula samples was validated. These results indicate that the present LC-MS/MS method combined with the MAE extraction is satisfactorily good for the analysis of DCD in infant formula samples.

\section{ACKNOWLEDGMENTS}

Financial support from the Science Technology Department of Zhejiang Province (China; 2011C23120) and AQSIQ (Beijing, China; 2011IK248) is gratefully acknowledged. The Ministry of Science and Technology (MOST, Beijing, China; 201410070) and the National
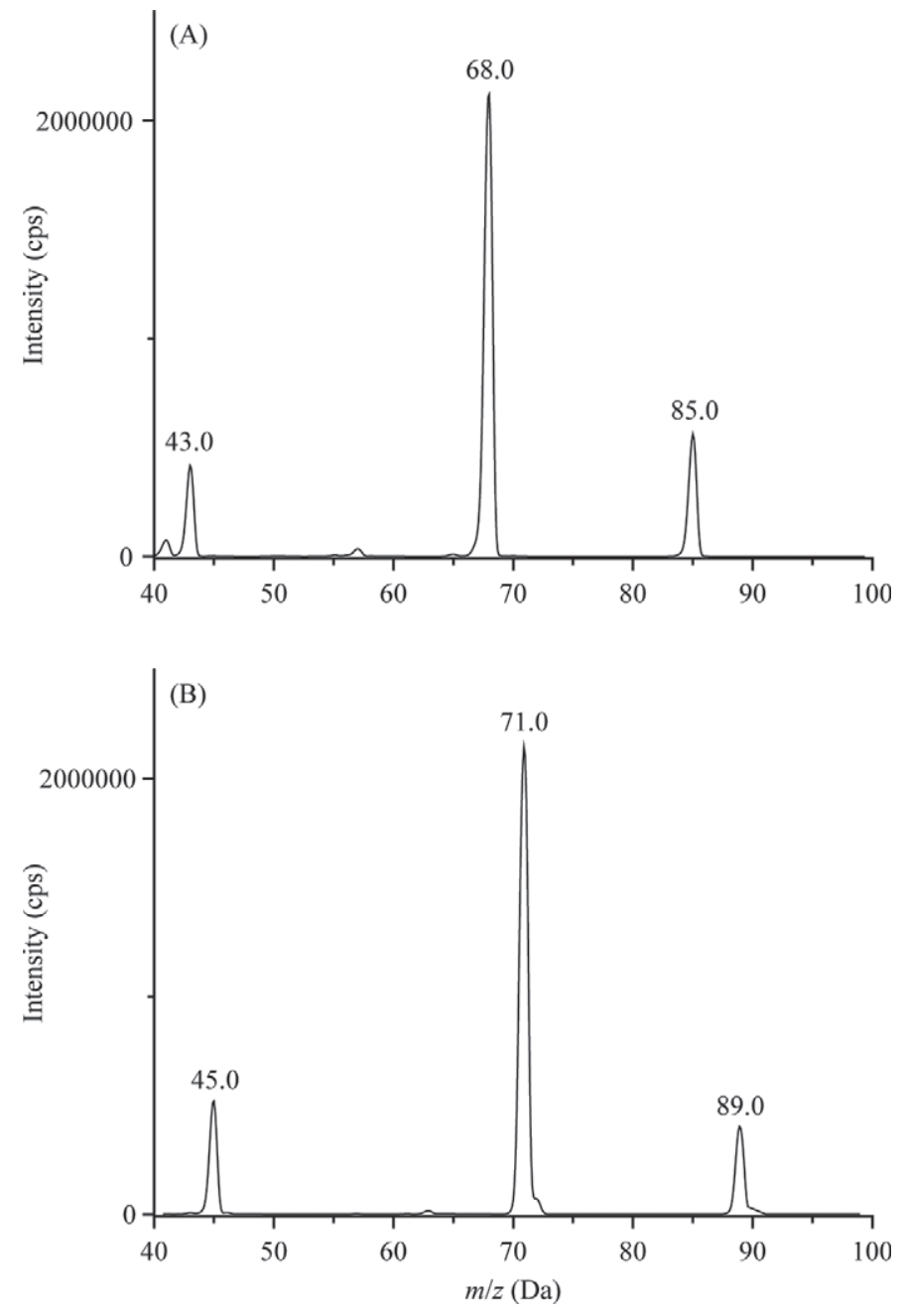

Figure 3. Electrospray ionization-tandem mass spectrometry (ESIMS/MS) product scan spectrum of (A) dicyandiamide (DCD) and (B) ${ }^{15} \mathrm{~N}_{4}$-labeled DCD $\left({ }^{15} \mathrm{~N}_{4}\right.$-DCD $)$.

Natural Science Foundation of China (NSFC, Beijing, China; 21207102) are gratefully acknowledged.

\section{REFERENCES}

Camel, V. 2000. Microwave-assisted solvent extraction of environmental samples. TrAC Trends Anal. Chem. 19:229-248.

Clough, T. J., H. J. Di, K. C. Cameron, R. R. Sherlock, A. K. Metherell, H. Clark, and G. Rys. 2007. Accounting for the utilization of a $\mathrm{N}_{2} \mathrm{O}$ mitigation tool in the IPCC inventory methodology for agricultural soils. Nutr. Cycl. Agroecosyst. 78:1-14.

Han, C., C. Liu, Y. Zhou, B. Xia, Z. Zhu, X. Lei, and Y. Shen. 2011. Microwave-assisted extraction and determination of cyanuric acid residue in infant formula samples by liquid chromatography-tandem mass spectrometry. Food Chem. 127:875-879.

MacMahon, S., T. H. Begley, G. W. Diachenko, and S. A. Stromgren. 2012. A liquid chromatography-tandem mass spectrometry method for the detection of economically motivated adulteration in protein-containing foods. J. Chromatogr. A 1220:101-107.

Nagumo, Y., K. Tanaka, K. Tewari, K. Thiraporn, T. Tsuchida, T. Honma, N. Ohtake, K. Sueyoshi, Y. Takahashi, and T. Ohyama. 
2009. Rapid quantification of cyanamide by ultra-high-pressure liquid chromatography in fertilizer, soil or plant samples. J. Chromatogr. A 1216:5614-5618.

Niessen, W. M. A., P. Manini, and R. Andreoli. 2006. Matrix effects in quantitative pesticide analysis using liquid chromatography-mass spectrometry. Mass Spectrom. Rev. 25:881-899.

Qin, J., K. Chao, and M. S. Kim. 2013. Simultaneous detection of multiple adulterants in dry milk using macro-scale Raman chemical imaging. Food Chem. 138:998-1007.

Schwarzer, C., and K. Haselwandter. 1996. Rapid quantification of the nitrification inhibitor dicyandiamide in soil samples, nutrient media and bacterial cell-free extracts. J. Chromatogr. A 732:390393.

Wakelin, S. A., T. J. Clough, E. M. Gerard, and M. O'Callaghan. 2013. Impact of short-interval, repeat application of dicyandiamide on soil $\mathrm{N}$ transformation in urine patches. Agric. Ecosyst. Environ. $167: 60-70$.

Wang, H., G. Li, Y. Zhang, H. Chen, Q. Zhao, W. Song, Y. Xu, H. Jin, and L. Ding. 2012. Determination of triazine herbicides in cereals using dynamic microwave-assisted extraction with solidification of floating organic drop followed by high-performance liquid chromatography. J. Chromatogr. A 1233:36-43. 